\title{
Plaquette Resonating-Valence-Bond Ground State of $\mathrm{CaV}_{4} \mathrm{O}_{9}$
}

\author{
Kazuo Ueda and Hiroshi Kontani \\ Institute for Solid State Physics, University of Tokyo, \\ 7-22-1 Roppongi, Minato-ku, Tokyo 106, Japan \\ Manfred Sigrist and Patrick A. Lee \\ Department of Physics, Massachussetts Institute of Technology, \\ Cambridge, Massachussetts 02139
}

(September 6, 2018)

A theoretical model is presented to explain the spin gap observed for $\mathrm{CaV}_{4} \mathrm{O}_{9}$. The underlying lattice of the $1 / 5$ depleted square lattice favors a formation of plaquette resonating valence bond state. Inclusion of the frustrating second neighbor interaction enhances this tendency, leading to a quantum disordered state of a two dimensional spin-1/2 Heisenberg model with a sufficiently big spin gap compatible with experiments.

PACS numbers: 75.10.Jm, 75.30-m, 75.40-s

Quantum disordered phases with a spin gap are of great current interest. This topic has gained additional momentum by Anderson's proposal of the resonating valence bond (RVB) state in the undoped parent materials of high temperture superconductivity [1]. Some of the typical examples with spin gaps are the spin-1 antiferromagnetic Heisenberg chain [2], the double chain spin- $1 / 2$ Heisenberg model [3], the spin-1/2 Heisenberg antiferromagnet on a Kagomé lattice [4], and Kondo spin liquid phase of the Kondo lattice model at half-filling [5].

Recently a new system with a spin gap was found experimentally by Taniguchi et al. for $\mathrm{CaV}_{4} \mathrm{O}_{9}$ [6]. The spin gap observed by magnetic susceptibility and nuclear magnetic resonance (NMR) measurements is $\Delta / k_{\mathrm{B}}=$ $107 \mathrm{~K}$. In this paper we propose that the underlying lattice of the $1 / 5$-depleted square lattice of $\mathrm{CaV}_{4} \mathrm{O}_{9}$, see Fig.1, favors a new type of spin disordered phase which may be called as plaquette resonating-valencebond (PRVB) state.

Each vanadium ion occupies a crystallographycally equivalent site and is surrounded by a pyramid of oxygens. First let us discuss the electronic state of this cluster $\left(\mathrm{VO}_{5}\right)^{2-}$. In this configuration the vanadium ion is in $\mathrm{V}^{4+}$ with one $d$-electron. Since $\mathrm{V}^{4+}$ is surrounded by a pyramid of oxygen ions, the $d$-electron is in either $d_{x z}$ or $d_{y z}$ orbital. This two-fold degeneracy is lifted by a small Jahn-Teller distorsion whose existence is reported in $[6]$ but its details are not yet clear [7]. However for our discussion of the spin gap, the details are not important because $\mathrm{V}^{4+}$ has a magnetic moment of spin- $1 / 2$ for which single ion anisotropy is absent.

The couplings between the spins on the vanadium ions are mediated by superexchange via the oxygen orbitals. The nearest neighbor vanadium ions share an edge of the square of oxgens (edge sharing), while the next nearest neighbor pairs share an oxgen at a corner (corner sharing). Superexchanges between the spins are possible through the hybridization with the $p_{z}$ orbitals of these oxgens. Since the number of paths for the edge sharing and the corner sharing is two and one, respectively, we expect $J_{1}$ (edge sharing) $\cong 2 J_{2}$ (corner sharing). Thus an appropriate model for $\mathrm{CaV}_{4} \mathrm{O}_{9}$ is the spin- $1 / 2$ Heisenberg model on the $1 / 5$-depleted square lattice

$$
\mathcal{H}=J_{1} \sum_{n . n .} \mathbf{s}_{i} \cdot \mathbf{s}_{j}+J_{2} \sum_{n . n . n .} \mathbf{s}_{i} \cdot \mathbf{s}_{j} .
$$

The magnitude of the exchange couplings may be estimated ifrom the susceptibility data [6]. At high temperatures it is reasonable to assume a Curie-Weiss behavior and the Weiss constant is given by $k_{\mathrm{B}} \theta=\frac{1}{3} s(s+$ 1) $\left(z_{1} J_{1}+z_{2} J_{2}\right)$, where $s$ is the spin quantum number, $z_{1}$ is the number of nearest neighbors and $z_{2}$ the number of next nearest neighbors. For the $1 / 5$-depleted square lattice $z_{1}=z_{2}=3$. From the intersect of the inverse susceptibility with the temperature axis it is estimated as $\theta=220 \mathrm{~K}$ [8]. Under the assumption of $J_{1}=2 J_{2}$, we obtain $J_{1} / k_{\mathrm{B}} \sim 200 \mathrm{~K}$.

To understand specific features of the $1 / 5$-depleted square lattice let us consider a cluster of four spins on a plaquette. The Hamiltonian of this system is

$\mathcal{H}_{\text {plaquette }}=J_{1}\left(\mathbf{s}_{1}+\mathbf{s}_{3}\right) \cdot\left(\mathbf{s}_{2}+\mathbf{s}_{4}\right)+J_{2}\left(\mathbf{s}_{1} \cdot \mathbf{s}_{3}+\mathbf{s}_{2} \cdot \mathbf{s}_{4}\right)$.

This Hamiltonian is readily diagonalized as is shown in Table I, where $S_{13}\left(S_{24}\right)$ is the spin quantum number of $\mathbf{s}_{1}+\mathbf{s}_{3}\left(\mathbf{s}_{2}+\mathbf{s}_{4}\right)$ and $S$ is the total spin quantum number.

Table I Eigenstates of a plaquette

\begin{tabular}{|l|c|c|c|c|c|c|}
\hline \hline$S_{13}$ & 0 & 1 & 0 & \multicolumn{3}{|c|}{1} \\
$S_{24}$ & 0 & 0 & 1 & \multicolumn{3}{|c|}{1} \\
\cline { 5 - 7 }$S$ & 0 & 1 & 1 & 0 & 1 & 2 \\
\hline$E_{g}$ & $-\frac{3}{2} J_{2}$ & $-\frac{1}{2} J_{2}$ & $-\frac{1}{2} J_{2}$ & $-2 J_{1}+\frac{1}{2} J_{2}$ & $-J_{1}+\frac{1}{2} J_{2}$ & $J_{1}+\frac{1}{2} J_{2}$ \\
\hline \hline
\end{tabular}

It is seen that the ground state of the plaquette is a singlet and the first excited state is a spin triplet with a spin gap of $J_{1}$ for $J_{1} / 2>J_{2}$. At $J_{1}=2 J_{2}$ the spin neutral excitation crosses with the spin triplet excitation. 
To proceed further let us first discuss a simplified Heisenberg model on the 1/5-depleted square lattice with only nearest neighbor couplings $\left(J_{2}=0\right)$. This model is very interesting in its own right, because the ground state of this model is probably disordered having no long range order or it is at least very close to a quantum phase transition, in spite of the fact that the lattice is bipartite and completely two-dimensional. For this lattice structure, there are two topologically inequivalent bonds. One type of bonds which will be called as plaquette-bonds hereafter form a plaquette covering of all spins, Fig. 2. We introduce an exchange coupling, $J$ for this type of bonds. The other type of bonds, dimer-bonds, form a complete dimer covering of the lattice and we use $J^{\prime}$ for the exchange coupling of this type. Although our final aim is to discuss the ground state of the model with $J=J^{\prime}$, for the time being we consider $J$ and $J^{\prime}$ as independent parameters. In comparison, all bonds are equivalent in the square lattice. A dimer or plaquette covering is possible in this case too. However, in contrast to the above lattice it is not unique and it is known that the square lattice favors an antiferromagnetically ordered ground state.

We begin our discussion from the limit $J \gg J^{\prime}$. In this limit, as we have discussed (Table I with $J_{1}=J$ and $J_{2}=0$ ), four spins on each plaquette form a singlet ground state which has a character of resonating valence bond [1]. The ground-state energy of this state per spin is $E_{\mathrm{PRVB}}=-\frac{1}{2} J$. In the other limit, $J^{\prime} \gg J$, the global ground-state is a collection of dimer singlets, $E_{\text {dimer }}=-\frac{3}{8} J^{\prime}$. For $J \approx J^{\prime}$, another possible ground state is one with antiferromagnetic long-range order, since the lattice is bipartite. The energy of the classical Néel state is $E_{\text {Néel }}=-\frac{1}{8} J^{\prime}-\frac{1}{4} J$. These energies are plotted in Fig. 3. The three states discussed here may be considered as the simplest variational states for three different phases. At this level, the spin disordered phase, either the dimer phase or the PRVB phase, has a lower energy than the Néel state. Concerning the two singlet phases it should be mentioned that they are different phases although the global symmetry properties are the same for the two phases. It can be shown that the wave function of the dimer singlet state has no overlap with that of the PRVB state based on the fact that the two wave functions have different transformation properties under the reflection with respect to the dimer-bonds: odd for the dimer singlet while even for the PRVB singlet. Thus, the two states cannot be continuously connected by tuning $J$ and $J^{\prime}$ from one to the other limit.

It is necessary to improve the estimate of the groundstate energies for the three phases. For the singlet phases we can use perturbation theories. Let us take the example of the PRVB state. When a dimer-bond is introduced, polarization processes from the singlets on both ends of the bond should be included. Polarization energy per bond may be calculated by the second order perturbation as $-(43 / 576)\left(J^{\prime 2} / J\right)$. Similar perturbation caluculation is also possible from the other limit of dimer singlet. The results are summarized as

$$
\begin{aligned}
E_{\mathrm{PRVB}} & =-\frac{1}{2} J\left[1+\frac{43}{576}\left(\frac{J^{\prime}}{J}\right)^{2}\right], \\
E_{\text {dimer }} & =-\frac{3}{8} J^{\prime}\left[1+\frac{1}{4}\left(\frac{J}{J^{\prime}}\right)^{2}\right] .
\end{aligned}
$$

These energy are also plotted in Fig.3.

For the Néel ordered phase a possible improvement is obtained by the linear spin wave theory [9]. Extension of the linear spin wave theory to the present case is rather complicated but straightforward. The ground-state energy per spin in this approximation is

$$
E_{\text {Néel }}\left(J=J^{\prime}\right)=-J\left[\frac{3}{2} s^{2}+0.325248 s\right],
$$

where $s=1 / 2$ for the present model. At $J=J^{\prime}$ the energy obtained by the spin wave theory, $-0.5376 \mathrm{~J}$, is very close to that of the PRVB state estimated by the second order perturbation, $-0.5373 J$. In the spin wave theory it is possible to calculate the reduction of magnetic moment by the zero point fluctuations of the magnons, $\delta s=0.288$ which is nearly $50 \%$ larger than the reduction for the square lattice, $\delta s=0.197$ and amounts to $58 \%$ of the magnitude of spin. The linear spin wave theory shows that the Néel order survives at this level but is on the verge of quantum phase transition. In view of the fact that the linear spin wave theory has a tendency to favor the Néel order [10], more careful treatments are necessary.

If there is a transition from the disordered phase to the Néel phase it would be a second order transition. In this case the spin gap vanishes at the transition point. Therefore the critical point may be estimated by examining at the spin gap in the disordered phases. A spin-triplet excitation in a plaquette is mobile. It can hop to a neighboring plaquette with an effective hopping matrix element, $J^{\prime} / 6$, to a second neighbor plaquette with $-J^{\prime 2} / 36 J$ and to a third neighbor plaquette with $-J^{\prime 2} / 216 J$. The polarization energies for the bonds connected to the triplet are different form the polarization energy in the ground state, $-(289 / 3456)\left(J^{\prime 2} / J\right)$. From these results the spin gap is calculated as

$$
\Delta_{\mathrm{PRVB}}=J\left[1-\frac{2}{3}\left(\frac{J^{\prime}}{J}\right)-\frac{111}{864}\left(\frac{J^{\prime}}{J}\right)^{2}\right] .
$$

Similar second order perturbation gives the spin gap for the dimer phase,

$$
\Delta_{\text {dimer }}=J^{\prime}\left[1-\frac{J}{J^{\prime}}-\frac{1}{2}\left(\frac{J}{J^{\prime}}\right)^{2}\right] .
$$

Within the second order perturbation theory, the spin gap vanishes at $\left(J^{\prime} / J\right)_{c}=1.215$ from the PRVB side and at $\left(J / J^{\prime}\right)_{c}=0.732$ from the dimer side. These points are shown by the dots in Fig. 3. This result suggests that in the narrow region between these critical points, antiferromagnetic long range order would exist. However, the spin gap remains finite at $J=J^{\prime}$ within the present perturbation theory. 
An alternative way to estimate the critical points is a cluster mean field theory. We explain the method by a simple example. For the PRVB state we consider a cluster with four spins on a plaquette under the influence of molecular fields coming from the dimer-bonds,

$\mathcal{H}_{\mathrm{CMF}}=J\left(\mathbf{s}_{1}+\mathbf{s}_{3}\right) \cdot\left(\mathbf{s}_{2}+\mathbf{s}_{4}\right)-J^{\prime} \sigma\left(s_{1}^{z}-s_{2}^{z}+s_{3}^{z}-s_{4}^{z}\right)$.

In the cluster mean field theory the average of a spin is determined by the self-consistence equation, $\sigma=\left\langle s_{1}^{z}\right\rangle$. This four spin problem can be solved analytically and the critical value is obtained as $\left(J^{\prime} / J\right)_{c}=3 / 4$. When a bigger cluster of 16-spins is used the critical value increases to $\left(J^{\prime} / J\right)_{c}=0.8044$. One can use a similar cluster mean field approximation for the dimer singlet. The smallest cluster of two spins gives $\left(J / J^{\prime}\right)_{c}=1 / 2$. The next one of 8 -spin cluster gives $\left(J / J^{\prime}\right)_{c}=0.5378$. From both sides the critical value increases as the cluser size becomes larger. Therefore we may consider $\left(J^{\prime} / J\right)_{c}=3 / 4$ and $\left(J / J^{\prime}\right)_{c}=1 / 2$ as the lower limits for the critical points, if any. However, unfortunately, cluster size is not big enough to perform a reliable finite size scaling to determine the existence or absence of the Néel phase.

All treatments discussed above suggest that the spin$1 / 2$ Heisenberg model on the $1 / 5$-depleted lattice with only nearest neighbor couplings has the spin disordered ground state in the wide region of parameter space. Only in a narrow region around the crossing point between the PRVB phase and the dimer phase, there is a possibility of the antiferromagnetic long range order. The recent quantum Monte Carlo simulations by Katoh and Imada [11] suggests that there is a spin gap of $\Delta=0.11 \mathrm{~J}$ for the model with $J=J^{\prime}$ consistent with the perturbation results.

Let us return to the original model, Eq.(1), keeping the different exchanges for the dimer-bonds and the plaquette bonds. It should be noted that the second nearest neighbor coupling is frustrating for the Néel order. It may be best illustrated by considering the cluster mean field theory. Again we consider the smallest cluster for the PRVB singlet,

$$
\begin{aligned}
\mathcal{H}_{\mathrm{CMF}}= & J\left(\mathbf{s}_{1}+\mathbf{s}_{3}\right) \cdot\left(\mathbf{s}_{2}+\mathbf{s}_{4}\right)+J^{\prime \prime}\left(\mathbf{s}_{1} \cdot \mathbf{s}_{3}+\mathbf{s}_{2} \cdot \mathbf{s}_{4}\right) \\
& -\left(J^{\prime}-2 J^{\prime \prime}\right) \sigma\left(s_{1}^{z}-s_{2}^{z}+s_{3}^{z}-s_{4}^{z}\right) .
\end{aligned}
$$

Since the eigenstates without the molecular field are completely determined by quantum numbers listed in Table I, the critical value is obtained analytically as $\left(\frac{J^{\prime}-2 J^{\prime \prime}}{J}\right)_{c}=\frac{3}{4}$. Thus we may conclude that the model for $\mathrm{CaV}_{4} \mathrm{O}_{9}$ has the quantum disordered ground state with safe margin.

We can extend the second order perturbation theory for the spin gap of the PRVB state with the frustating exchange coupling, for which we obtain

$$
\begin{aligned}
\Delta_{\mathrm{PRVB}}= & J\left\{1-\frac{2}{3}(x-2 y)-\frac{1}{54}\left(7 x^{2}-10 x y+10 y^{2}\right)\right. \\
& -\frac{1}{12} \frac{2 x^{2}-3 x y+4 y^{2}}{2-y}+\frac{7}{18} \frac{(x-y)^{2}+y^{2}}{3-y}
\end{aligned}
$$

$$
\left.-\frac{1}{12} \frac{x^{2}+2 y^{2}}{3-2 y}-\frac{5}{72} \frac{(x-y)^{2}+y^{2}}{4-y}\right\},
$$

where $x=J^{\prime} / J$ and $y=J^{\prime \prime} / J$. From this result it is seen that the gap increases as $J^{\prime \prime}$ increases. This behavior is illustrated in Fig. 4 for the case $J=J^{\prime}$. A similar (8)behavior is observed in the expansion around the dimer limit which gives,

$$
\Delta_{\text {dimer }}=J^{\prime}\left\{1-x^{-1}\left(1-\frac{3}{2} y\right)-\frac{1}{8} x^{-2}\left(4-4 y+9 y^{2}\right)\right\} .
$$

It is clear that quantitative results of the perturbation theory is questionable at $J=J^{\prime}=J_{1}$, which is seen by the difference between the values of the spin gap for the model with only nearest neighbor coupling. $\Delta=0.205 J_{1}$ is obtained by the PRVB perturbation theory on one hand and by the Quantum Monte Carlo simulations, $\Delta=0.11 J_{1}$ on the other hand [11]. When we use the results of the Quantum Monte Carlo simulations the magnitude of the spin gap is too small compared with the experimental value. The present perturbation result shows that the spin gap increases significantly when we include the frustrating next nearest neighbor exchange of the order of $J_{2}=J_{1} / 2$, which may lead to a reasonable value of the spin gap compared with the experiments.

In conclusion the spin-1/2 Heisenberg model on the $1 / 5$-depleted square lattice is presented as a theoretical model for the spin gap of $\mathrm{CaV}_{4} \mathrm{O}_{9}$. It is shown that the $1 / 5$ depleted square lattice is favorable for the quantum spin disordered phase, which may be characterized as the PRVB singlet. When the frustrating exchange for the corner sharing bonds is included it is possible to explain the large spin gap observed experimentally.

\section{ACKNOWLEDGMENTS}

It is our great pleasure to acknowledge fruitful discussions with Masatoshi Sato, Hirokazu Tsunetsugu, Yoshio Kitaoka, and Maurice Rice. This work is financilly supported by a Grant-in-Aid ifrom the Ministry of Education, Science and Culture of Japan. We acknowledge financial support from Swiss Nationalfonds (M.S.; (No. 8220-037229) and from M.I.T. Science Partnership Fund and from the NSF (P.L. and M.S.; DMR-90-22933).

A significant part of this work was done during the stay in Aspen Center for Physics of K.U. and M.S.

[1] P.W. Anderson, Science 235, 1196 (1987).

[2] F.D.M. Haldane, Phys. Lett. 93A, 464 (1983); Phys. Rev. Lett. 50, 1153 (1983). 
[3] S.P. Strong and A.J. Millis, Phys. Rev. Lett. 69, 2419 (1992).

[4] C. Zeng and V. Elser, Phys. Rev. B 42, 8436 (1990).

[5] H. Tsunetsugu, Y. Hatsugai, K. Ueda, and M. Sigrist, Phys. Rev. B 46, 3175 (1992).

[6] S. Taniguchi, T. Nishikawa, Y. Yasui, Y. Kobayashi, M. Sato, T. Nishioka, M. Kontani, and K. Sano, J. Phys. Soc. Jpn, 64, 2758 (1995).

[7] Another possibility is the $d_{x y}$ orbital since Vanadium ion is shifted from the basal plane towards inside of the pyramid of oxgens. Even if this is the case the following discussions about the superexchange remains qualitatively the same with the use of $p_{x}$ and $p_{y}$ orbitals.

[8] M. Sato, private communication.

[9] P.W. Anderson, Phys. Rev. 86, 694 (1952); R. Kubo, ibid. 87, 568 (1952).

[10] One typical example is the double-layer Heisenberg model; T. Matsuda and K. Hida, J. Phys. Soc. Jpn, 59, 2223 (1990); K. Hida, ibid. 61, 1013 (1992).

[11] N. Katoh and M. Imada, private communication.

FIG. 1. A model for $\mathrm{CaV}_{4} \mathrm{O}_{9}$ of the spin-1/2 Heisenberg model with the nearest neighbor (solid lines) and the next nearest neighbor (broken lines) exchange interactions. The dot dashed lines show the unit cell of the $1 / 5$ depleted square lattice.

FIG. 2. Spin 1/2 Heisenberg model on the 1/5-depleted square lattice with the nearest neighbor exchange interactions. Topologically there are two different type of bonds: plaquette-bonds $J$ and dimer-bonds $J^{\prime}$.

FIG. 3. Ground state energies for the dimer singlet, the PRVB siglet, and the Néel ordered phase. The horizontal axis is $\tilde{x}$ defined by $J / J^{\prime}=\tilde{x} /(1-\tilde{x})$. The points where the spin gap vanishes are denoted by the dots. $E_{S W}$ is the energy estimated by the linear spin wave theory at $J=J^{\prime}$

FIG. 4. The spin gap as a function of frustrating exchange coupling, $y=J^{\prime \prime} / J$. For $J^{\prime \prime} / J>0.354$ the spin gap deviates from Eq.(10) because the minimum of the spectrum is different from $(\pi, \pi)$. 


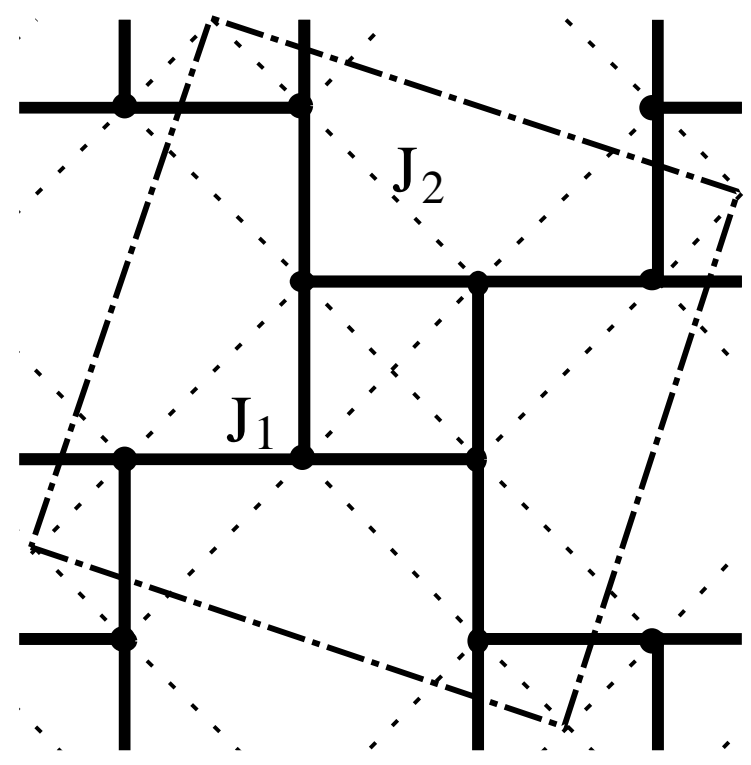




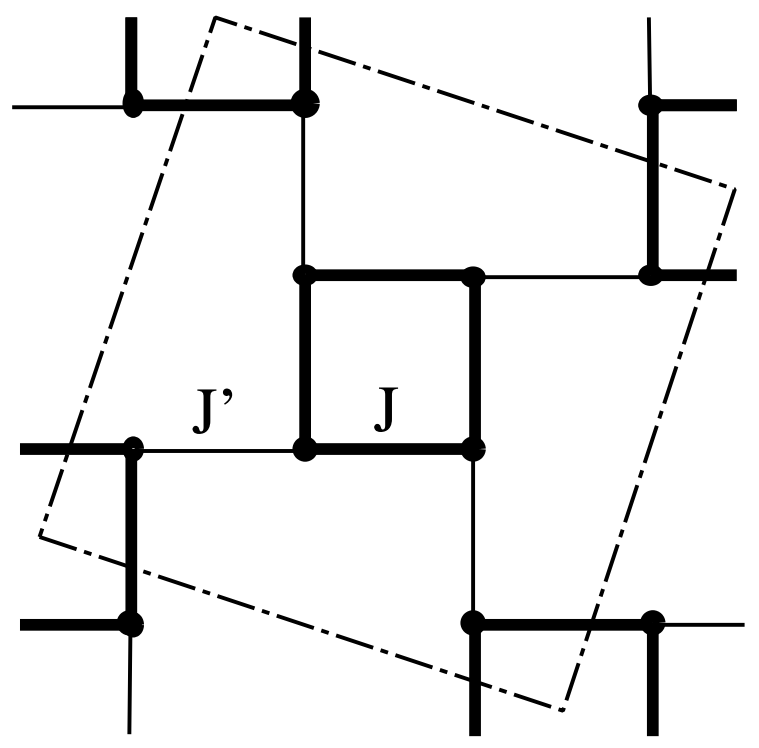




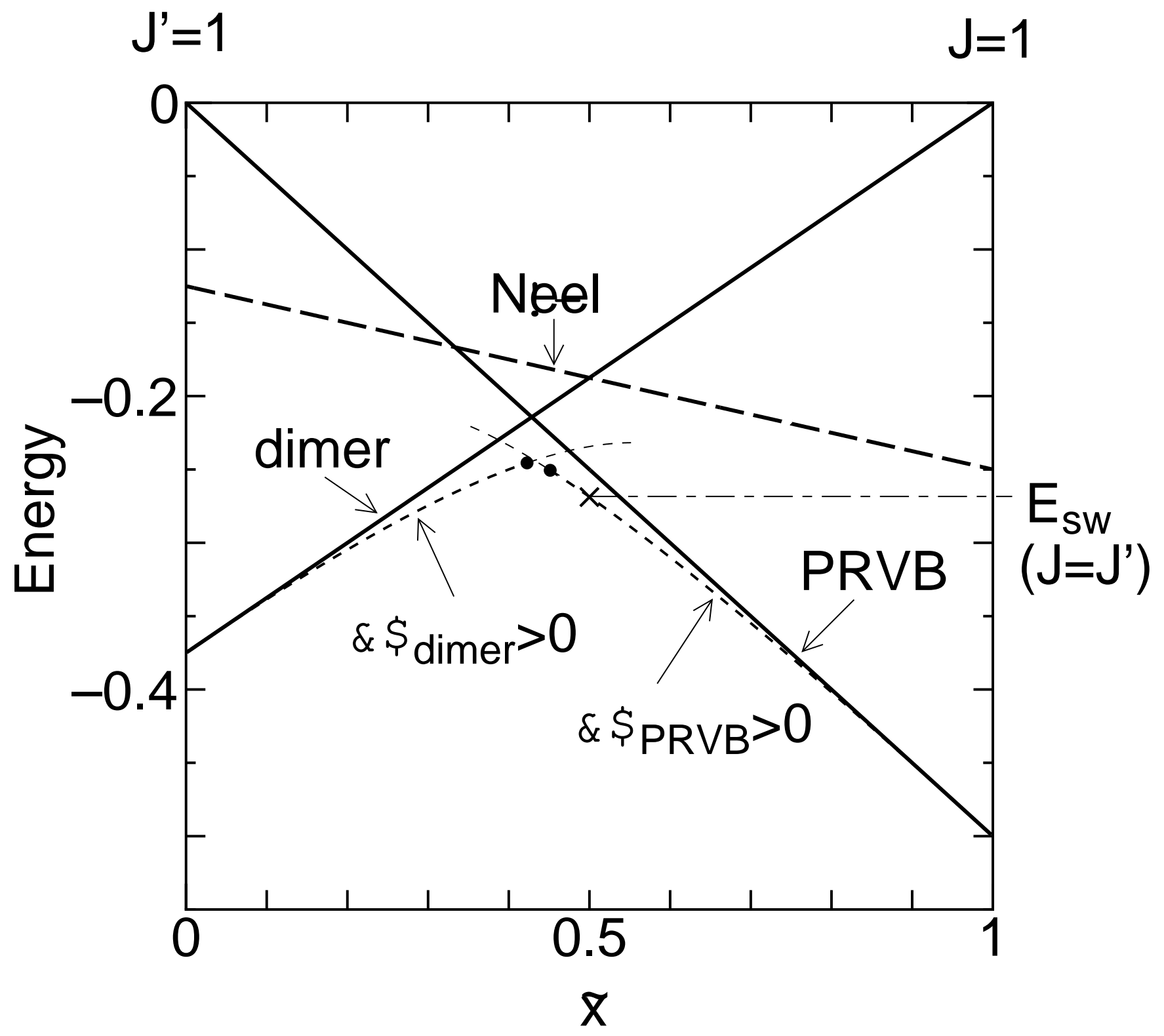




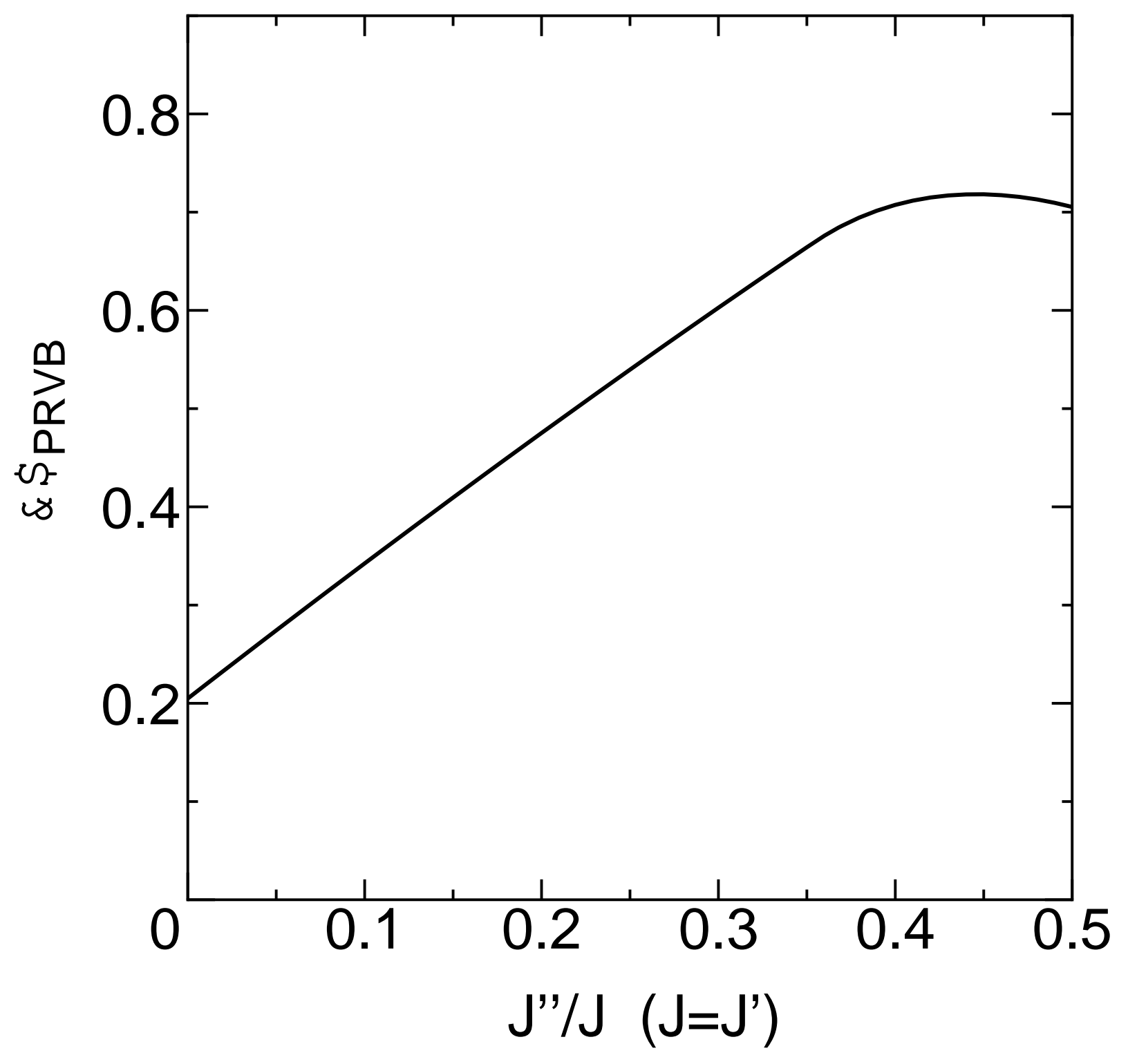

